\title{
Sensitivity Analysis of Microstructure Parameters and Mechanical Strength during Consolidation of Cemented Paste Backfill
}

\author{
Xuebin Qin (D), Pai Wang, Lang Liu $(\mathbb{D}$, Mei Wang $(\mathbb{D}$, and Jie Xin \\ School of Electrical and Control Engineering, Xian University of Science and Technology, 58 Yanta Rd, Xian, Shaanxi, China \\ Correspondence should be addressed to Xuebin Qin; qinxb@xust.edu.cn
}

Received 10 February 2018; Revised 6 April 2018; Accepted 15 April 2018; Published 23 May 2018

Academic Editor: Giovanni Minafò

Copyright (C) 2018 Xuebin Qin et al. This is an open access article distributed under the Creative Commons Attribution License, which permits unrestricted use, distribution, and reproduction in any medium, provided the original work is properly cited.

\begin{abstract}
Parameter sensitivity is an important part of the quantitative model uncertainty, which helps to effectively identify the key parameters, reduce the uncertainty of the parameters, and then improve the efficiency of parameter optimization. In order to accurately and intuitively analyze the influence of the microscopic parameters and the mechanical response of consolidation process of cemented paste backfill (CPB), a method is used to characterize the geometric and morphological features of the CPB. In this paper, digital image processing technology is used to propose a method for the identification and quantitative analysis of microscopic pore images based on CPB. The pore images on the microscopic scale of CPB are obtained by the microscopic analysis of SEM images, binarization, denoising, and other operations; further, several microscopic parameters are calculated on the pore image, such as the porosity, uniformity coefficient, fractal dimension, probability entropy, and other quantitative parameters, realizing quantitative analysis of pore images. The microstructure of pore images of CPB is extracted under different curing times and then the parameter sensitivity between the microstructure parameters and the mechanical response based on the finite-difference method is analyzed. Set microstructure parameter software of consolidation process of CPB is developed based on this idea, which can be used to identify microscopic pore images and analyze the morphology quantitatively. The microcosmic parameters of $\mathrm{CPB}$ with strong sensitivity are uniformity coefficient, average shape coefficient, sorting coefficient, fractal dimension, average length of long axis, average pore area, weighted probability entropy, pore number, and porosity. The sensitivity of the remaining parameters is relatively low. Therefore, the CPB is preferably used in the strength testing process. The method provides a new method for the quantitative analysis of parameter sensitivity on the microscale of CPB.
\end{abstract}

\section{Introduction}

In recent years, with the gradual reduction of shallow mineral resources in the earth, deep mining of mineral resources already tends to normalize. Mining filling technology has become more and more important $[1,2]$. With the development of cement filling technology and the need of mine environmental protection, the tailings discharged from the concentrator become the main aggregates of cementing and filling in the mine gradually. With different water-cement ratio, different curing times of $\mathrm{CPB}$ have a direct impact on the mechanical properties of the relationship. Many scholars use different research methods and techniques to study the factors affecting the strength of tailings CPB. Kesimal A studied the relationship between the strength of the deboning copper-lead-zinc tailings and the paste and found that the grain size distribution of the tailings sand had a great influence on the strength of the CPB [3]. Fall et al. studied the effect of curing temperature on the strength of tailings $\mathrm{CPB}$ [4]. G Xiu et al. experiments were carried out under different material ratios; the work reveals the microscopic chemical reaction mechanism of the tailings and studied the influence on the stability of the CPB [5]. Chun Liu et al. extracted the basic parameters of pores such as porosity, fractal dimension, and uniformity coefficient by the manual threshold on a single SEM image and analyzed the relationship between the microstructure and mechanics of rock $[6,7]$. Serge Outllet et al. studied SEM images of different samples of $\mathrm{CPB}$ and analyzed the pore structure. This method is used to estimate three pore structure parameters: total porosity, pore 
size distribution, and spatial curvature of pores [8]. Many researchers have made deep research on the composition ratio, stabilization process, and mechanical strength of tailing CPB $[9,10]$. It is very important to analyze the microscopic parameter sensitivity, for example, the characterization of the microscopic parameters of the $\mathrm{CPB}$, how to establish the primary and secondary relationship of the parameters, the parameters of the inversion calculation, and the accurate calculation of the model. At present, there are few sensitive parameters in the microstructure of $\mathrm{CPB}$. The authors investigated sensitivity analysis of landslide parameters [11], sensitivity analysis of hydrological model parameters [12], and sensitivity analysis of geotechnical parameters of slope engineering [13]. The intelligent technique is a combination of the artificial neural network and particle swarm optimization, which is used to predict the unconfined compressive strength of CPB [14]. The Curing Under Applied Pressure System (CUAPS) is used to simulate CPB placement and curing processes and then to assess the strength development of the samples under consolidated and drained conditions via the application of external pressure on the fill sample top $[15,16]$. The effects of curing conditions on changes in CPB microstructure and corresponding unconfined compressive strength were assessed using both an improved CUAPS system and conventional plastic molds [17, 18]. A predictive model is proposed for unconfined compressive strength of CPB by self-weight consolidation $[19,20]$. In the paper, a method of mechanical response and parameter sensitivity analysis is proposed based on microscale image processing technology of CPB.

This paper takes the $\mathrm{CPB}$ of different curing times as the research object; the image processing technology is used to extract the micropore image by the indoor microtest. According to measure indexes, such as total pore area, uniformity coefficient, fractal dimension, average pore area, number of pores, maximum pore area, and weighted probability entropy, geometric features and morphology of pores are characterized. The influence of the microcosmic parameters for the sensitivity of the mechanical response of $\mathrm{CPB}$ is analyzed. It provides an accurate and direct quantitative method for the relationship between the pore development structure and the mechanical response of the CPB. The core goal of this paper is to characterize the macro mechanical characteristics of $\mathrm{CPB}$ by microscopic parameters. The microcosmic parameters can be used to characterize the microscopic mechanical response and predict macroscopic mechanical characteristics of $\mathrm{CPB}$.

\section{Materials and Methods}

2.1. Material Component. The main material for the filling test is the full tailings of the Xiang Furnace Shan Tungsten mine, the strength grade of 325\# type composite Portland cement (PCC), and the urban tap water. The basic performance of the tailings is tested in the experiment and the distribution curve of the grain size is obtained. As shown in Figure $1, d_{10}$ indicates that the grain size of the tailings is $3.630 \mu \mathrm{m}$. $\mathrm{d}_{50}$ is $52.667 \mu \mathrm{m}, \mathrm{d}_{90}$ is $132.095 \mu \mathrm{m}$, and

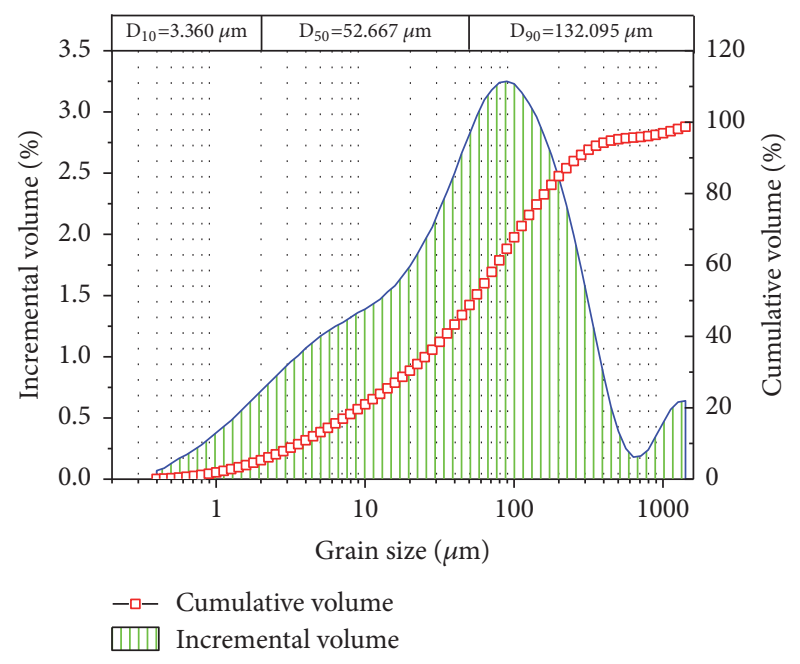

FIGURE 1: Tailing grain size distribution curve.

specific surface area is $631 \mathrm{~m} 2 / \mathrm{kg}$, The uniformity coefficient of the grain size of tailings is 20.82. The best grade of tailings particles should be consistent with Thabo equation. Generally, it should be between 4 and 6 .

It is known from the grain size curve of the tailings. The content of coarse grains in the test tailings is less, which belongs to the type of relatively missing coarse grains. The natural gradation of tailings belongs to the relative discontinuous gradation. The measurement results of the specific gravity, bulk density, porosity, and natural rest angle of the tailings are listed in Table 1. The main chemical composition and mass fraction of tailings were obtained by $\mathrm{X}$-ray fluorescence spectrum analysis of tailings. It is shown in Table 2.

\subsection{Test Process}

2.2.1. Sample Preparation. In the experiment, four identical samples were made (one was a spare sample and three were test samples) according to the calculation of the cement sand ratio and required Portland cement weight, and then they were weighed. According to the ratios in Table 3, the tailings and cement were weighed and mixed evenly, and the sample was prepared by adding city tap water (the slurry mass fraction was $72 \%$ ). A small agitator was used for more than 5 min until the CPB was stirred evenly. A layer of Vaseline was applied to the circular cast iron test mold, which had a diameter of $50 \mathrm{~mm}$ and a height of $100 \mathrm{~mm}$. The CPB was loaded in the test mold in three layers. Each layer was then compacted by vibration. The $\mathrm{CPB}$ remained still for $24 \mathrm{~h}$ after being loaded. The surface of the test sample was smoothed by scraping, and the mold was removed. The test samples were properly labeled and placed in a constant-temperature, constant-humidity curing box at a temperature of $20 \pm 1^{\circ} \mathrm{C}$ and humidity of $95 \pm 1 \%$.

2.2.2. Uniaxial Compression Strength Test. Each test sample was removed, and its height and diameter were measured with a Vernier caliper 3, 7, 14, 28, and 56 days after curing. A 
TABLE 1: Test of specific gravity, loose bulk density, dense bulk density, porosity, and natural rest angle.

\begin{tabular}{lccccc}
\hline material & Specific gravity & $\begin{array}{c}\text { Loose bulk } \\
\text { density } \\
\left(\mathrm{t} / \mathrm{m}^{3}\right)\end{array}$ & $\begin{array}{c}\text { Dense bulk } \\
\text { density } \\
\left(\mathrm{t} / \mathrm{m}^{3}\right)\end{array}$ & $\begin{array}{c}\text { Porosity } \\
(\%)\end{array}$ \\
\hline Full tailings & 2.992 & 1.392 & 1.955 & 34.659 \\
\hline
\end{tabular}

TABLE 2: Full-tail grading composition.

\begin{tabular}{lcccccccc}
\hline chemical composition & $\mathrm{TFe}$ & $\mathrm{SiO}_{2}$ & $\mathrm{Al}_{2} \mathrm{O}_{3}$ & $\mathrm{MgO}$ & $\mathrm{CaO}$ & $\mathrm{S}$ & $\mathrm{WO}_{3}$ & others \\
\hline content (\%) & 9.45 & 48.22 & 5.01 & 2.96 & 12.68 & 2.75 & 0.055 & 18.875 \\
\hline
\end{tabular}

TABLE 3: Experimental design.

\begin{tabular}{lccc}
\hline No. & Slurry concentration/\% & Gelling agent & cement-sand ratio \\
\hline 1 & 72 & OPC & \\
2 & 72 & OPC & $1: 12$ \\
3 & & OPC & \\
\hline
\end{tabular}

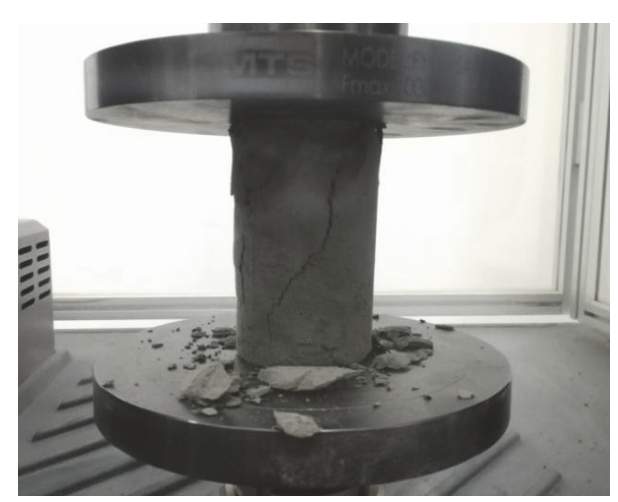

Figure 2: Uniaxial compressive strength test of cemented tailings backfill.

computer-controlled $20-\mathrm{kN}$ pressure machine was employed to apply pressure at a constant rate of $1 \mathrm{~mm} / \mathrm{min}$ until the test sample failed, as shown in Figure 2. The data were then collated to compute the uniaxial compressive strengths of the test pieces, which were then averaged to obtain the result.

2.3. Preparation of SEM Samples. In this study, SEM samples were fabricated. The method is a modern detection technology, which has the characteristics of high resolution, large magnification time, and wide field of view of threedimensional images. Consequently, the samples had to be dried and gilded to obtain a true and clear observation.

The CPB was selected at different curing times for preparation of the SEM test samples. First, the middle part of the $\mathrm{CPB}$ was located, and a double-sided blade-wire-saw coated with Vaseline was used to cut a $10 \mathrm{~mm} \times 10 \mathrm{~mm} \times 10 \mathrm{~mm}$ roughcast cube with a $1.5 \mathrm{~mm}$ border. A sharp backfill paste steel knife was then used to cut the blank and create a fresh cross section, baring a complete natural structural surface. A $5 \mathrm{~mm} \times 5 \mathrm{~mm} \times 5 \mathrm{~mm}$ block was used for observation under the electron microscope. For this, the fresh surface had to be as flat as possible, with all disturbance particles removed by a rubber suction bulb.

\section{Extraction of Micropore Image and Quantitative Analysis}

3.1. Extraction of Micropore Image. Based on the method of kernel fuzzy clustering, we used the image processing technology to extract the microimage of the CPB in the SEM image obtained by the indoor microtest. First, the method of kernel fuzzy clustering was used to divide the SEM images into five clusters. The darkest category in the cluster image was used as the adaptive extracted pore image. The micropore image was treated by binarization, and the binary pore image was obtained.

3.1.1. Pore Image Extraction Based on the Kernel Fuzzy C Means Clustering Method. The kernel fuzzy C means clustering algorithm is an iterative dynamic clustering algorithm. It is applied to clinical diagnosis, pathology analysis, image compression, image recognition, and so on. In this work, a fuzzy clustering algorithm was used to extract the pore image on the microscale of the CPB. Based on many experiments, the microscale images of the CPB were divided into five types, and the darkest clustering image was used as the pore image. The principle of the algorithm is the minimization of the target function. In the experiment, the kernel function fuzzy clustering algorithm was used to obtain the pore image [21]. The target function is as follows:

$$
\mathrm{J}(U, V)=\sum_{i=1}^{N} \sum_{k=1}^{c} u_{i k}^{m}\left(1-K\left(x_{i}, v_{k}\right)\right)
$$

where $U=\left\{u_{i k}\right\}_{c \times N}$ is a matrix of fuzzy membership degree. $V=\left\{v_{k}\right\}_{c \times 1}$ represents the cluster center matrix. $N$ represents the total number of sample points. $C$ represents the number of clusters. And $x_{i}(i=1,2, \ldots, N)$ represents the sample set; $u_{i k}$ indicates that $x_{i}$ belongs to the membership degree of cluster k. $v_{k}(k=1,2, \ldots, c)$ represents the cluster center. Kernel function $K\left(x_{i}, v_{k}\right)=\exp \left(-\left\|x_{i}-v_{k}\right\|^{2} / \sigma\right)$ is a Gauss kernel function. The expression of $u_{i k}$ is

$$
u_{i k}=\frac{1}{\sum_{l=1}^{c}\left(\left(1-K\left(x_{i}, v_{k}\right)\right) /\left(1-K\left(x_{i}, v_{l}\right)\right)\right)^{1 /(m-1)}}
$$




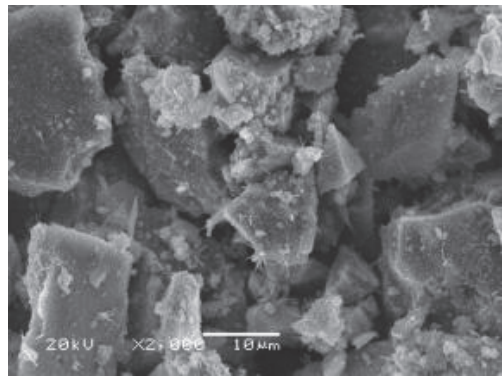

(a) Original image

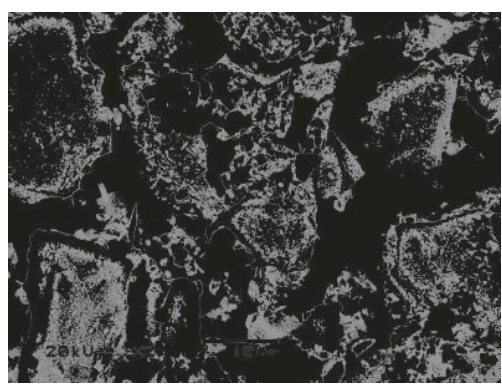

(d) Fairly dark

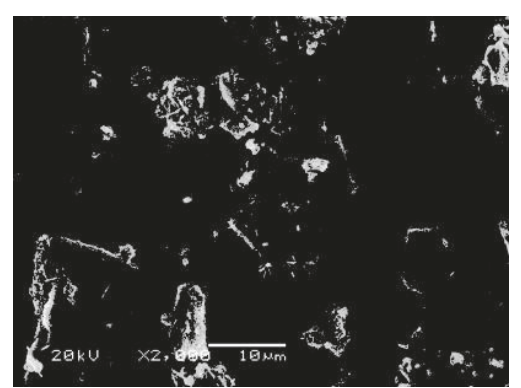

(b) Bright

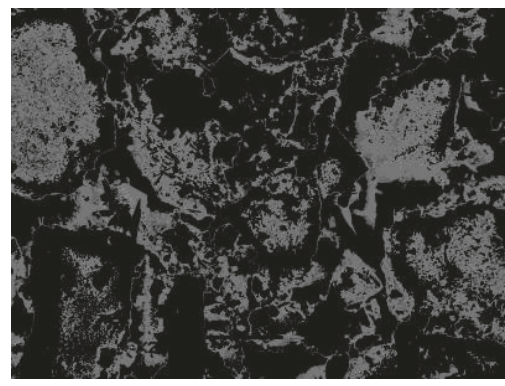

(e) Dark

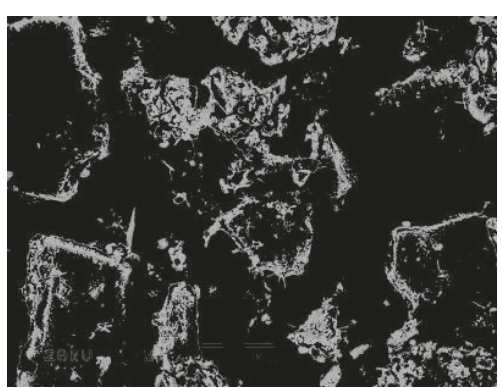

(c) Fairly bright

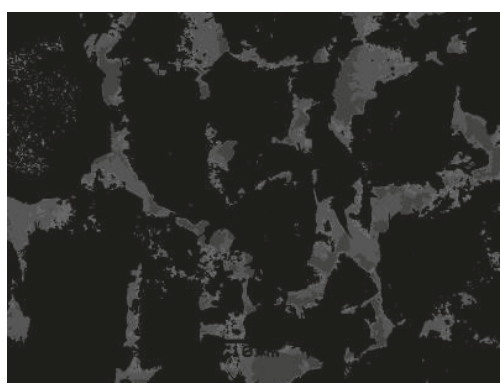

(f) Darkest

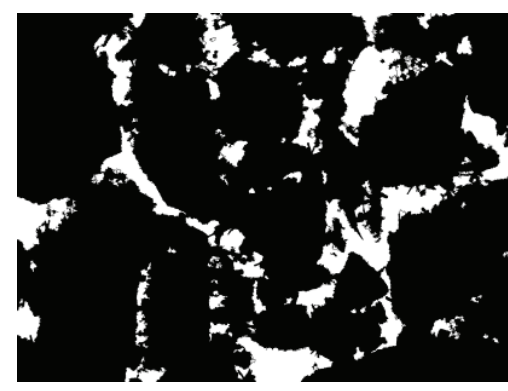

(g) Binary image of pore

FIGURE 3: Clustering results of pore images for CPB based on the fuzzy clustering method.

The expression of cluster center $v_{k}$ is

$$
v_{k}=\frac{\sum_{i=1}^{N} u_{i k}^{m} K\left(x_{i}, v_{k}\right) x_{i}}{\sum_{i=1}^{N} u_{i k}^{m} K\left(x_{i}, v_{k}\right)}
$$

The steps of the algorithm are as follows.

Step 1. Set the number of clusters $C(c>=2)$, fuzzy exponent $m$, and the iterative terminating condition $\varepsilon$.

Step 2. Initialize cluster center $v_{k}^{(0)}$.

Step 3. Set cycle counter $b=0$.

Step 4. Update membership function $u_{i k}^{(b)}$ according to formula (2).

Step 5. Update cluster center function $v_{k}^{(b+1)}$ according to formula (3).

Step 6. If $\max _{1 \leq k \leq c}\left|v_{k}^{(b)}-v_{k}^{(b+1)}\right|<\varepsilon$ or when the number of iterations is greater than the threshold for a given number of iterations, then the cyclic iteration is terminated. Otherwise, set $b=b+1$ and jump to Step 4 .

In this experiment, SEM images of CPB with different curing times were used, and the curing times were $3 \mathrm{~d}, 7 \mathrm{~d}$, $14 \mathrm{~d}, 28 \mathrm{~d}$, and $56 \mathrm{~d}$. The pore image was extracted based on the fuzzy clustering method. The SEM images were divided into five clusters: bright, fairly bright, fairly dark, dark, and darkest. The darkest image was extracted from the cluster as a pore image because the pore is in the darkest region of the image. The parameters of the clustering algorithm are as follows: $m=2, c=5, \delta=2, x_{i}$ is the gray value of the gray image, and $\varepsilon$ is an iterative error, $\varepsilon \in(0,1)$. As shown in Figure 3 , the fuzzy clustering method is used for classification. The picture is an SEM image of the 3D (day) curing times. The picture is divided into five clusters. (a) is the original image, and (b) through (f) are five cluster images: bright, fairly bright, fairly dark, dark, and darkest. From the diagram in (f), it can be seen that this type of image objectively reflects the distribution of pores on a microscale, so this type of image is taken as a pore image. The image $(\mathrm{g})$ is a binary image of the image $(\mathrm{f})$. 


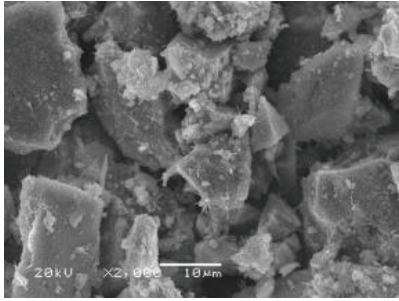

(a) Original image (3d)

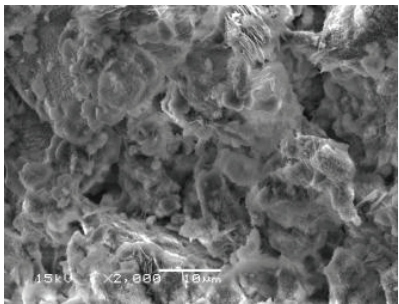

(e) Original image (14d)

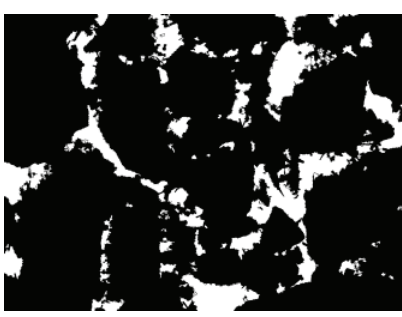

(b) Binary image of pore

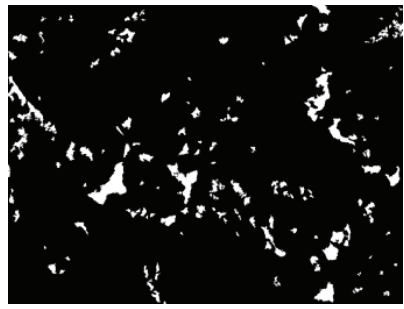

(f) Binary image of pore

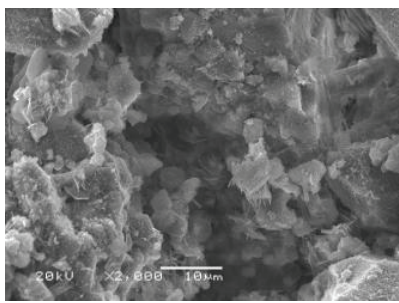

(i) Original image (56d)

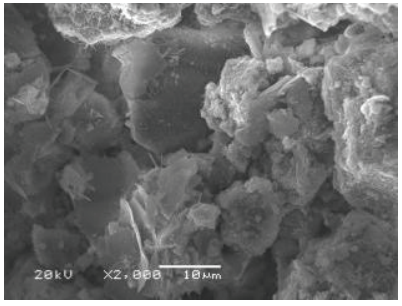

(c) Original image $(7 \mathrm{~d})$

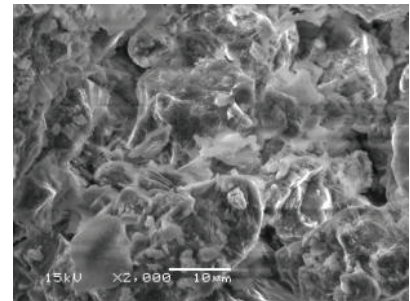

(g) Original image (28d)

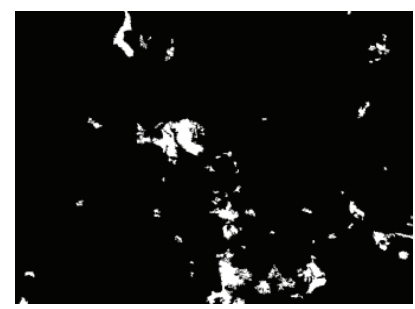

(j) Binary image of pore

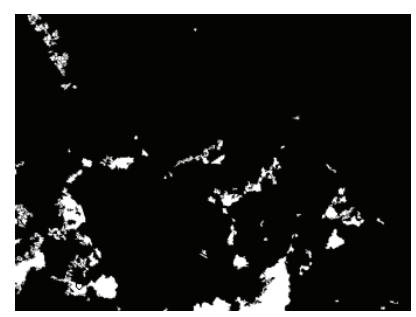

(d) Binary image of pore

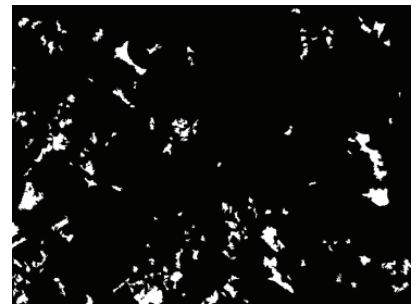

(h) Binary image of pore

FIGURE 4: SEM image and corresponding pore binary image under different curing times.

3.1.2. Accurate Extraction of Pore Images. To extract the exact pore area, there are many small heterojunction areas in Figure 3(f). In the process of software processing, areas less than 40 pixels were removed as noise, and the remaining binary image as a pore was reversed. Fine connections were removed by morphological treatment. Figure 4 shows the binary image when the miscellaneous area is removed. The curing ages of (b) through (f) are $3 \mathrm{~d}, 7 \mathrm{~d}, 14 \mathrm{~d}, 28 \mathrm{~d}$, and $56 \mathrm{~d}$, respectively, and the SEM scanning images and corresponding pore binary images are shown.

3.2. Quantitative Parameter Analysis. In this study, the pore image was extracted from the SEM image of CPB. To describe the microscopic pore characteristics of the CPB quantitatively, the relationship between the distribution, quantity, direction, size, and mechanical response of the pores is described quantitatively by many microscopic parameters.

The microscopic parameters included the pore number, total pore area, maximum pore area, average pore area, porosity, uniformity coefficient, sorting coefficient, curvature coefficient, roundness, fractal dimension, and weighted probability entropy.

According to the morphological and geometric characteristics of the binary pore images, the microcosmic index of the $\mathrm{CPB}$ was used in the quantitative analysis of the pores.

(1) Pore number represents the number and sizes of pores on the SEM image.
(2) Average length is defined as the length of a region by the Feret diameter, for all pores, which represents the accumulation of the long axis for each pore.

(3) Total pore area represents the sum of the total areas of all pores on the SEM image.

(4) Pore porosity represents the integrity of $\mathrm{CPB}$ pores, which is the ratio of the pore region to the total image area. The quantified index is a $2 \mathrm{D}$ parameter; it can indirectly reflect the changes of pore ratio in $3 \mathrm{D}$ space.

(5) Uniformity coefficient, $C_{u}$, represents the ratio of $d_{60}$ to $d_{10}$ :

$$
C_{u}=\frac{d_{60}}{d_{10}}
$$

where $d_{10}$ is the diameter of the pore image area when the cumulative area on pore image is $10 \% . d_{60}$ is the diameter of the pore image area when the cumulative area on the image is $60 \%$.

(6) The curvature coefficient represents continuity of the cumulative curve for the diameter of the pore image block.

$$
C_{c}=\frac{d_{30}^{2}}{\left(d_{60} * d_{10}\right)}
$$

where the meaning of $d_{10}$ and $d_{60}$ are the same as uniformity coefficient $C_{u}$, and $d_{30}$ is the diameter of the corresponding pore image block when the cumulative area is $30 \%$. 
(7) Sorting coefficient, $S_{c}$, is used to sort the pore image blocks in descending order by area. When the size of the pore area is uniform, the values, P25 and P75, are similar. Thus, $S_{c}$ is closer to 1 , and the other is larger than 1.

$$
S_{c}=\frac{p_{25}}{p_{75}}
$$

where $d_{25}$ and $d_{75}$ represent the diameter of the pore image block corresponding to the cumulative pore areas of $25 \%$ and $75 \%$, respectively.

(8) The fractal dimension of porosity is the quantitative index used to describe the CPB size distribution, which directly reflects the changing pore shape. The formula is defined as follows:

$$
D_{c}=\lim \frac{\ln N(r)}{\ln r} \quad r \longrightarrow 0,
$$

where the pore image was divided into small square grids. In our experiment, $r$ of each grid is $1,3,5$, 7 , etc. The maximum value of $r$ is a quarter of the image width. $N(r)$ denotes the number of pores in a square grid corresponding to $r$. A larger fractal dimension of porosity $\left(D_{c}\right)$ leads to a lower level of pore homogenization and a larger difference of size among pores.

(9) Weighted probability entropy is a quantitative parameter reflecting the regularity of structural units. It describes the overall CPB arrangement of pores at a microscopic scale. For the distribution in each small region, the computational formula of probability entropy, $h_{m}$, is defined as follows:

$$
h_{\mathrm{m}}=-\sum_{i=1}^{n} p_{i} \log _{n} p_{i}
$$

where $p_{i}$ is the frequency of a structural body in a certain directional region. $n$ is the interval of the orientation angles in the arrangement direction of structural units. The value, $\mathrm{hm}$, is between 0 and 1. A larger $h m$ leads to a more disordered arrangement of pores and lower regularity, and vice versa. In the experiment, given that $n=36$, every $10^{\circ}$ is a sector. The midpoint of the long axis is selected as the coordinate of the original point, with a horizontal $x$-axis and a vertical $y$-axis.

Owing to the size of each area block being different across the whole region, the contribution rate is also different. Thus, a new parameter is defined as the weighted probability entropy. All regional blocks on the pore image are subject to normalization, as shown in

$$
a_{i}=\frac{s_{i}}{\sum_{i=0}^{N-1} s_{i}}
$$

where there are $N$ image blocks on the pore image. $s_{i}$ is the area of the $i$ th region. $a_{i}$ is the contribution rate of the $i$ th region following normalization, which is regarded as the weighted value. The final weighted probability entropy is

$$
H_{m}=\sum_{i=0}^{N-1} a_{i} h_{m i}
$$

where $h_{m i}$ is the probability entropy of the $i$ th region, and $H_{m}$ is the overall probability entropy of the pore image.

(10) The definition of the circular degree $\mathrm{R}$ is as follows:

$$
R_{i}=\frac{4 \pi S}{L^{2}}
$$

where S represents the area of the block, and L represents the circumference of the block. If the error of the circular degree of a single pore is too large and is meaningless, the average circular degree is used to analyze the characteristics of the pore shape. Average shape coefficient is defined as follows:

$$
R=\sum_{\mathrm{i}=1}^{n} \frac{R_{i}}{n}
$$

where $n$ represents the number of pores. The larger $\mathrm{R}$ is, the more slippery the shape of the pores is, and vice versa.

\section{Sensitivity Analysis of Microcosmic Parameters and Strength of Pores}

4.1. Parameter Sensitivity Principle. From the SEM image of the CPB for different curing times, the pore image was extracted based on the fuzzy clustering method, and the corresponding microparameters were obtained. There are many factors affecting the mechanical properties of $\mathrm{CPB}$ owing to the pore microstructure: (1) the geometric parameters for the microcosmic characteristics of the $\mathrm{CPB}$, such as porosity, pore number, average pore area, average pore width, pore distribution characteristics, and pore fractal dimension; (2) characteristics of pore arrangement change in $\mathrm{CPB}$, such as probability entropy and rose map; (3) characteristics of pore morphological change in $\mathrm{CPB}$, such as circularity and the fractal dimension of the pore; and (4) distribution characteristics, such as uniformity coefficient, curvature coefficient, and separation coefficient. The relationship between the microscopic parameters and mechanical response is blindly constructed, and the size of the influence degree cannot be accurately evaluated. Therefore, sensitivity analysis is needed; the greater the sensitivity, the higher the degree of influence of the factor. To improve the accuracy of the relationship between the microparameters and mechanical response, the principle of priority is adopted for the sensitive factors. This work uses the dimensionless sensitivity.

Based on the finite-difference method, the microcosmic parameter sensitivity and mechanical response of the cemented filling body are analyzed. The finite-difference method is a numerical method to solve differential equations. The basic idea is that the continuous solution domain is replaced by a finite discrete node, and the approximate solution process is used to replace the partial differential equation with difference. The mathematical formula is as follows:

$$
\frac{d y}{d x}=\lim _{\Delta x \rightarrow 0} \frac{\Delta y}{\Delta x}=\lim _{\Delta x \rightarrow 0} \frac{f(x+\Delta x)-f(x)}{\Delta x}
$$

where $d y, d x$ are the differential of function and independent variable, respectively; $d y / d x$ is the first derivative of the function to the independent variable. 
TABLE 4: Microparameter calculation.

\begin{tabular}{|c|c|c|c|c|c|c|}
\hline \multirow{2}{*}{ No. } & \multirow{2}{*}{ Microcosmic parameters } & \multicolumn{5}{|c|}{ Curing times } \\
\hline & & $3 d$ & $7 \mathrm{~d}$ & $14 \mathrm{~d}$ & $28 \mathrm{~d}$ & $56 \mathrm{~d}$ \\
\hline 1 & Total area of image & 288000 & 288000 & 288000 & 288000 & 288000 \\
\hline 2 & Pore number & 75 & 84 & 78 & 51 & 52 \\
\hline 3 & Total pore area & 27227 & 37935 & 32581 & 32279 & 52809 \\
\hline 4 & Maximum pore area & 4876 & 6254 & 6869 & 5519 & 10770 \\
\hline 5 & Average pore area & 363.02 & 451.61 & 417.71 & 632.92 & 1015.57 \\
\hline 6 & Average length of long axis & 35.22 & 44.55 & 40.21 & 48.26 & 48.75 \\
\hline 7 & porosity & 0.09 & 0.13 & 0.11 & 0.11 & 0.18 \\
\hline 8 & Uniformity coefficient & 3.11 & 2.18 & 4.29 & 3.56 & 4.59 \\
\hline 9 & Sorting coefficient & 3.20 & 1.77 & 2.77 & 2.27 & 2.59 \\
\hline 10 & curvature coefficient & 0.82 & 1.19 & 0.91 & 1.09 & 0.92 \\
\hline 11 & Average shape coefficient & 0.26 & 0.20 & 0.26 & 0.25 & 0.29 \\
\hline 12 & fractal dimension & 0.94 & 0.95 & 0.95 & 0.91 & 0.93 \\
\hline 13 & Weighted probability entropy & 0.86 & 0.85 & 0.84 & 0.86 & 0.89 \\
\hline
\end{tabular}

TABLE 5: Sensitive values and contribution rate of 12 microscopic parameters on the pore image.

\begin{tabular}{lccc}
\hline No. & Microcosmic parameters & Sensitive value & Contribution rate (\%) \\
\hline 1 & Pore number & 4.70 & 7.44 \\
2 & Total pore area & 3.06 & 4.84 \\
3 & Maximum pore area & 1.00 & 1.58 \\
4 & Average pore area & 5.54 & 8.77 \\
5 & Average length of long axis & 6.31 & 9.99 \\
6 & porosity & 3.06 & 4.84 \\
7 & Uniformity coefficient & 11.00 & 17.46 \\
8 & Sorting coefficient & 7.58 & 12.00 \\
9 & curvature coefficient & 1.33 & 2.11 \\
10 & Average shape coefficient & 8.19 & 12.97 \\
11 & fractal dimension & 6.35 & 10.05 \\
12 & Weighted probability entropy & 5.02 & 7.95 \\
\hline
\end{tabular}

4.2. Calculation of Microparameter. The steps of calculating the sensitivity between the microscopic parameters and the mechanical response are as follows:

(1) The digital image processing software was used to analyze the SEM image of the CPB, and the related microparameters, as in Table 4, were obtained.

(2) The same microscopic parameters in each continuous curing times in Table 4 were subtracted, and four differences were taken for each parameter. The dimensionless processing was carried out, mapping the values to $0.1 \sim 1.1$, as shown in the following formula:

$$
x_{t}=\frac{x-x_{\min }}{x_{\max }-x_{\min }}+0.1
$$

(3) According to formula (13), the sensitivity and contribution rate of the influencing factors are calculated, as shown in Table 5.

4.3. Parameter Sensitivity Analysis. To analyze the sensitivity of the parameters, the finite-difference method was used to analyze the relationship between the multiple microscopic parameters and mechanical strength. The dimensionless treatment was used in the experiment because of the different dimensions between parameters. The parameter values of five different curing ages were obtained by the difference method, resulting in four different $\Delta x_{i}$. Then, the four difference values were mapped to $0.1 \sim 1.1$. The minimum difference was 0.1 , the maximum difference was 1.1, and the other difference was mapped. In the same way, the strength parameters of the mechanical response for five different curing times were calculated by differential operation; that is, four $\Delta y_{i}$ were obtained. Figure 5 is a mechanical strength map corresponding to five SEM images. To increase the recognition of the sensitive value mapping $\Delta y_{i}$ to $1 \sim 11$, the minimum difference was 1 , and the maximum difference was 11; the sensitivity of each parameter was defined as follows:

$$
s=\sum_{i=1}^{N}\left(\frac{\Delta y_{i}}{\Delta x_{i}}\right)^{2}
$$

where, in the experiment, $N=4$.

The size of the sensitivity of the microscopic parameters can be obtained from Table 5. The order of specific sensitivity from large to small is uniformity coefficient, average shape 


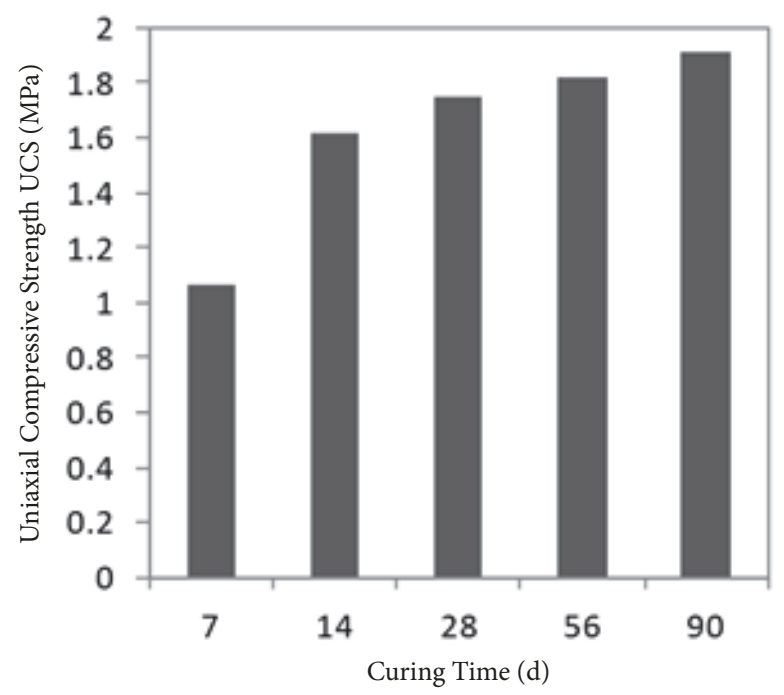

FIgURE 5: Uniaxial compressive strength of $\mathrm{CPB}$ at different curing times.

coefficient, sorting coefficient, fractal dimension, average length of long axis, average pore area, weighted probability entropy, pore number, and porosity. The cumulative contribution rate of the first nine microparameters' sensitivity was 91.11\%.

Therefore, when constructing the relationship between microscopic parameters and mechanical response, the nine parameters uniformity coefficient, average shape coefficient, sorting coefficient, fractal dimension, average length of long axis, average pore area, weighted probability entropy, pore number, and porosity are preferentially used.

\section{Conclusion}

(1) A computer intelligent recognition method for microscopic pore images of $\mathrm{CPB}$ was established. For the geometric and morphological structure of the $\mathrm{CPB}$, the quantitative description of the pore number, total pore area, maximum pore area, average pore area, long-axis average length, porosity, uniformity coefficient, sorting coefficient, curvature coefficient, roundness, fractal dimension, and weighted probability entropy index were proposed. This method can reduce human error and objectively reflect the microstructure of the $\mathrm{CPB}$.

(2) The results of sensitivity analysis showed that the contribution of the microparameters to the compressive strength of the $\mathrm{CPB}$ is different. The first nine parameters of the contribution rate are arranged in order from large to small: uniformity coefficient, average shape coefficient, sorting coefficient, fractal dimension, average length of long axis, average pore area, weighted probability entropy, pore number, and porosity. Therefore, in the process of strength testing, these parameters are preferred.

(3) To express the direction of the pores effectively, the concept of weighted probability entropy was proposed, and the contribution rate of the image region was different.
(4) Digital image processing technology provided an effective method for the quantitative analysis of cemented filling pores. It obtained good pore-quantizing results. When the three-dimensional image analysis technology of $\mathrm{CPB}$ is developed, the analysis of $\mathrm{CPB}$ can be realized more accurately and fully.

(5) This method is simple and easy, and its objective is to describe the microstructure of the $\mathrm{CPB}$.

\section{Data Availability}

The data used to support the findings of this study are available from the corresponding author upon request.

\section{Conflicts of Interest}

The authors declare that there are no conflicts of interest regarding the publication of this paper.

\section{Authors' Contributions}

Xuebin Qin and Pai Wang conceived and designed the experiments; Lang Liu and Mei Wang performed the experiments; Jie Xin analyzed the data; Lang Liu contributed SEM image; Xuebin Qin wrote the paper.

\section{Acknowledgments}

This work was supported by the National Natural Science Foundation of China (no. 51704229 and no. 51405381) and Key Scientific and Technological Project of Shaanxi Province (no. 2016GY-040).

\section{References}

[1] X. Dongsheng, "Discussion on reducing pressure technology of filling pipeline transport system in deep well," Express Information of Mining Industry, vol. 10, no. 2, pp. 25-28, 2007.

[2] Q.Zhang, Q. Liu, J.-W. Zhao, and J.-G. Liu, "Pipeline transportation characteristics of filling paste-like slurry pipeline in deep mine," The Chinese Journal of Nonferrous Metals, vol. 25, no. 11, pp. 3190-3195, 2015.

[3] A. Kesimal, B. Ercikdi, and E. Yilmaz, "The effect of desliming by sedimentation on paste backfill performance," Minerals Engineering, vol. 16, no. 10, pp. 1009-1011, 2003.

[4] M. Fall, J. C. Célestin, M. Pokharel, and M. Touré, "A contribution to understanding the effects of curing temperature on the mechanical properties of mine cemented tailings backfill," Engineering Geology, vol. 4, no. 10, pp. 397-413, 2010.

[5] G. Xiu, W. Dang, and Z. Liu, "Microstructure test and macro size effect on the stability of cemented tailings backfill," International Journal of Digital Content Technology and Its Applications, vol. 6, no. 14, pp. 387-397, 2012.

[6] C. Liu, B. Shi, J. Zhou, and C. Tang, "Quantification and characterization of microporosity by image processing, geometric measurement and statistical methods: Application on SEM images of clay materials," Applied Clay Science, vol. 54, no. 1, pp. 97-106, 2011. 
[7] C. Liu, C.-S. Tang, B. Shi, and W.-B. Suo, "Automatic quantification of crack patterns by image processing," Computers \& Geosciences, vol. 57, pp. 77-80, 2013.

[8] S. Ouellet and M Benzaazoua, "Characterization of cemented paste backfill pore structure using SEM and IA analysis," Bulletin of Engineering Geology and the Environment, vol. 67, no. 2, pp. 139-152, 2008.

[9] B. Ercikdi, H. Baki, and M. Izki, "Effect of desliming of sulphiderich mill tailings on the long-term strength of cemented paste backfill," Journal of Environmental Management, vol. 115, pp. 513, 2013.

[10] A. Ghirian and M. Fall, "Coupled thermo-hydro-mechanicalchemical behaviour of cemented paste backfill in column experiments," Engineering Geology, vol. 164, no. 3, pp. 195-207, 2013.

[11] S. Jiang and B.-P. Wen, "Analysis of sensitivity of landslide sliding mechanics parameters based on different methods," Journal of Engineering Geology, vol. 23, no. 6, pp. 1153-1162, 2015.

[12] X.-m. Song, F.-Z. Kong, and C.-S. Zhan, "Analysis of sensitivity of hydrological model parameter based on statistical theory," Journal of Advances in Water Science, vol. 23, no. 5, pp. 642-649, 2017.

[13] J.-j. Ge, "Sensitivity analysis of geotechnical parameter of railway slope engineering," Journal of Railway Construction, vol. 46, no. 11, pp. 107-110, 2014.

[14] C. Qi, A. Fourie, and Q. Chen, "Neural network and particle swarm optimization for predicting the unconfined compressive strength of cemented paste backfill," Construction and Building Materials, vol. 159, pp. 473-478, 2018.

[15] D. Landriault, "Paste backfill mix design for Canadian underground hard rock mining," in Proceedings of the 97th Annual General Meeting of the CIM Rock Mechanics and Strata Control Session, pp. 13-22, Halifax, Nova Scotia, Canada, 1995.

[16] M. Benzaazoua, T. Belem, and E. Yilmaz, "Novel lab tool for paste backfill," Canadian Mining Journal, vol. 127, no. 3, p. 31, 2006.

[17] E. Yilmaz, T. Belem, M. Benzaazoua, and B. Bussière, "Assessment of the modified CUAPS apparatus to estimate in situ properties of cemented paste backfill," Geotechnical Testing Journal, vol. 33, no. 5, 2010.

[18] E. Yilmaz, T. Belem, B. Bussière, and M. Benzaazoua, "Relationships between microstructural properties and compressive strength of consolidated and unconsolidated cemented paste backfill," Cement and Concrete Composites, vol. 33, pp. 702-715, 2011.

[19] T. Belem, E. Yilmaz, and M. Benzaazoua, "Predictive models for unconfined compressive strength of cemented paste backfills taking into account self-weight consolidation," in Proceedings of the 68th Canadian Geotechnical Conference, pp. 1-8, Quebec, Canada, 2015.

[20] E. Yilmaz, T. Belem, B. Bussière, M. Mbonimpa, and M. Benzaazoua, "Curing time effect on consolidation behaviour of cemented paste backfill containing different cement types and contents," Construction and Building Materials, vol. 75, pp. 99111, 2015.

[21] Z.-d. Wu, W.-x. Xie, and J.-p. Yu, "Sensitivity analysis of geotechnical parameter of railway slope engineering fuzzy cmeans clustering algorithm based on kernel method," in Proceedings of the 15th International Conference on Computational Intelligence and Multimedia Applications, pp. 49-54, 2003. 


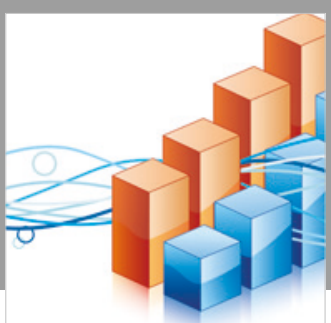

Advances in

Operations Research

\section{-n-m}
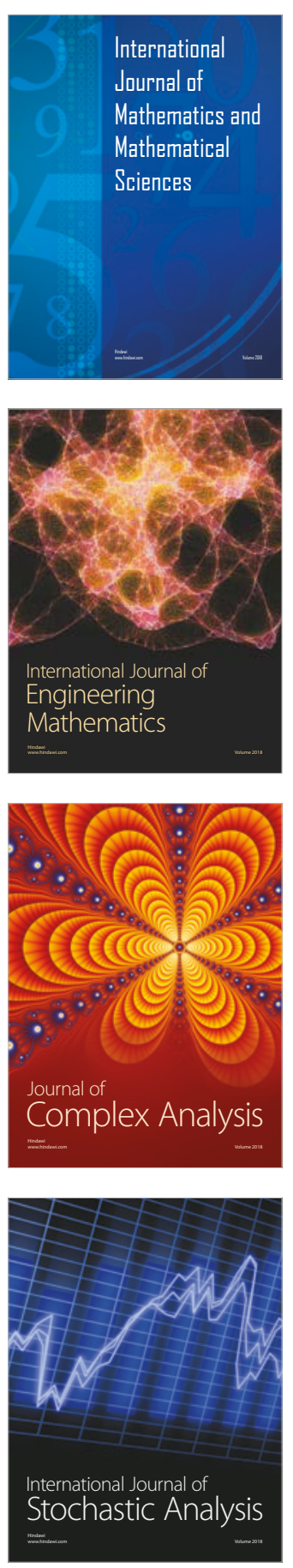
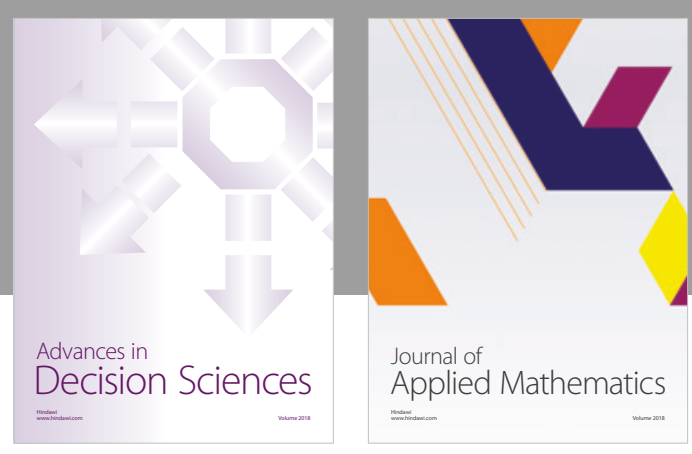

Journal of

Applied Mathematics
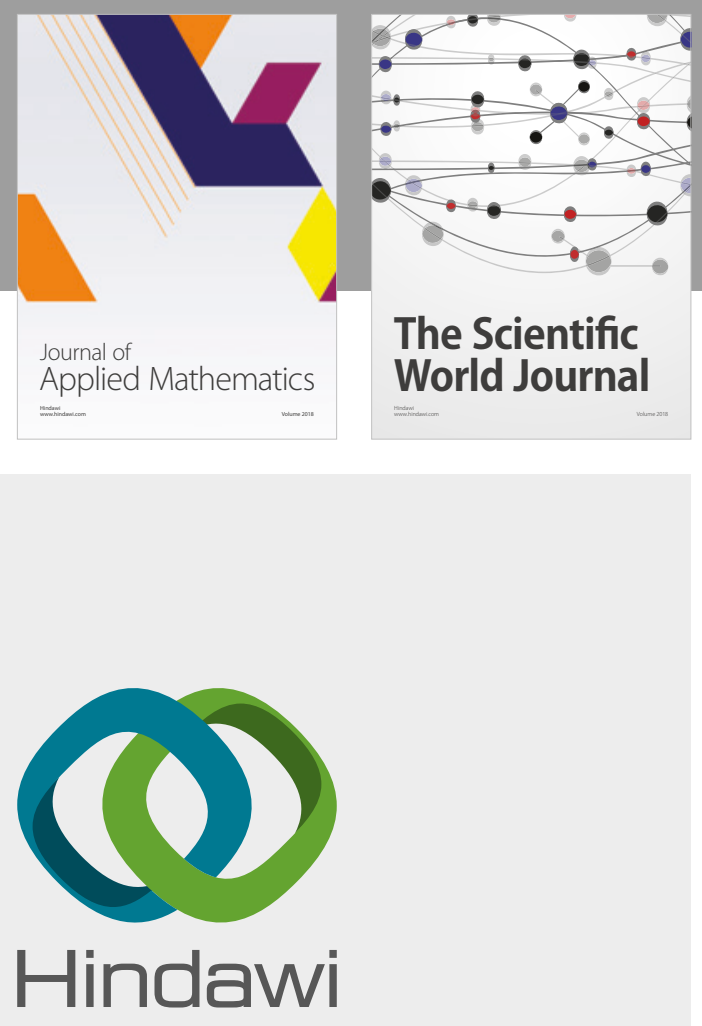

Submit your manuscripts at

www.hindawi.com

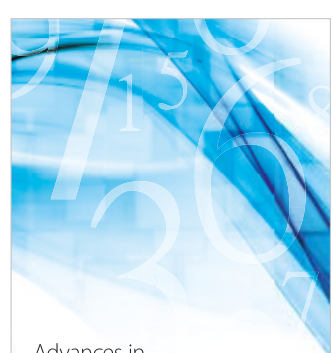

Advances in
Numerical Analysis
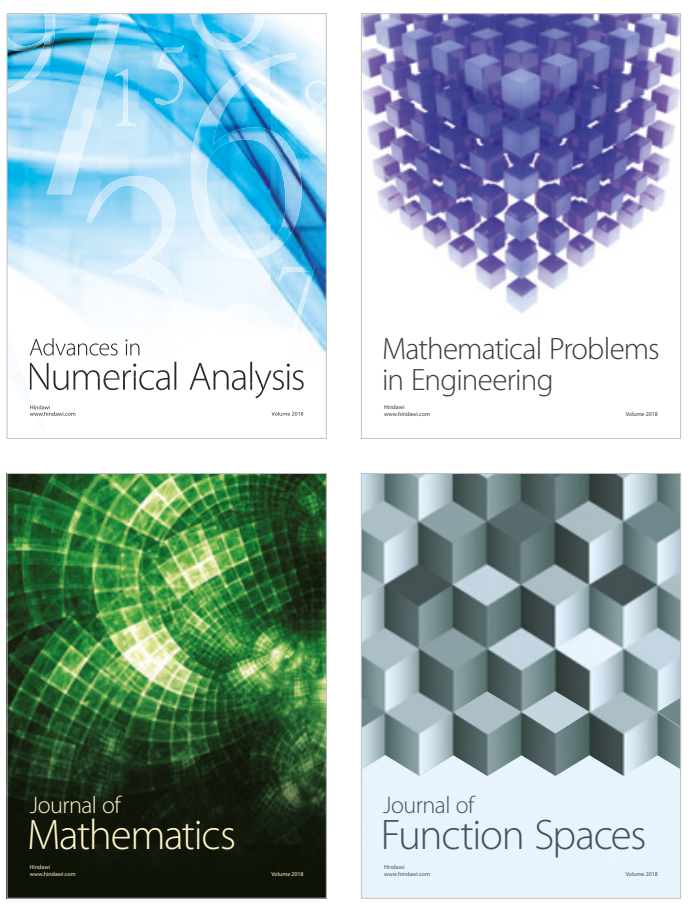

Mathematical Problems in Engineering

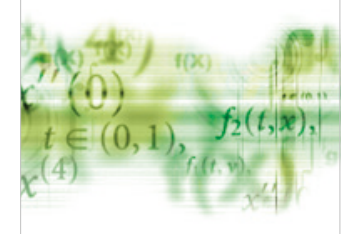

International Journal of

Differential Equations

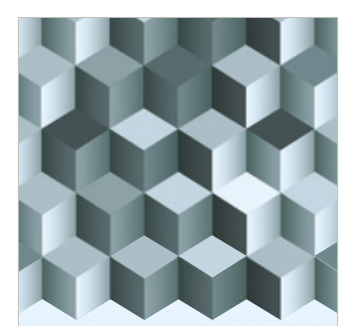

Journal of

Function Spaces

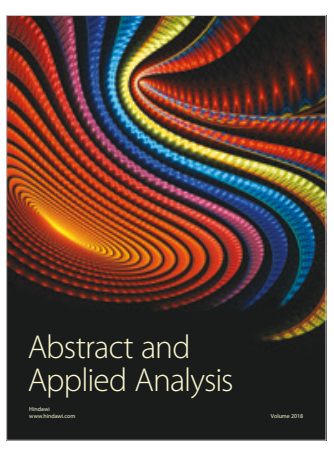

The Scientific

World Journal

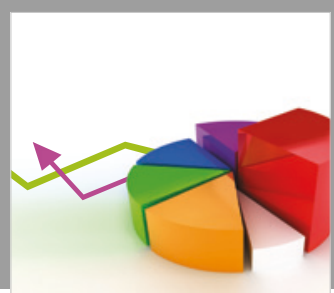

Journal of

Probability and Statistics
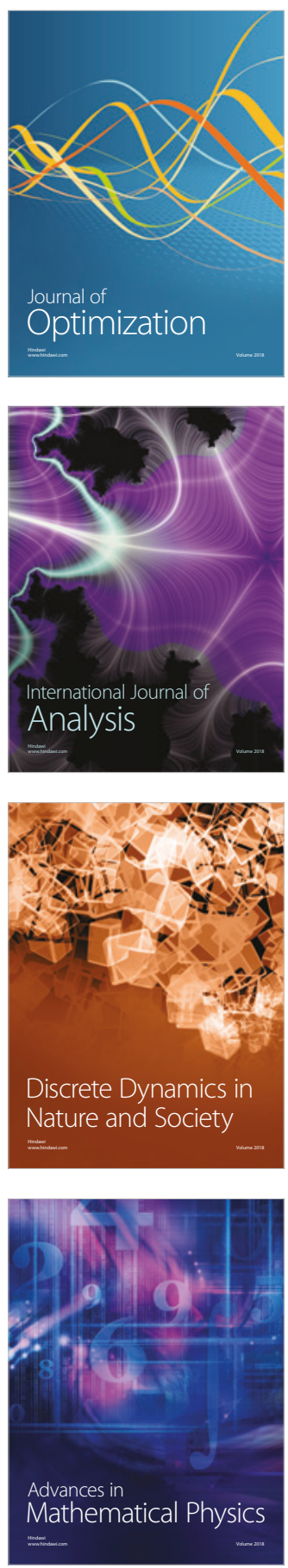